\title{
Erst am Anfang
}

Qualifizierte Regelbesetzung im OP Das Thema der personellen Mindestbesetzung in den Funktionsdiensten wird derzeit heftig diskutiert: Was ist angemessen, was ist unbedingt nötig und was ist fehlerinduziert? Wir stellen Ihnen einen Leitfaden für eine verantwortungsvolle und zielgerichtete Personalbesetzung im OP vor.

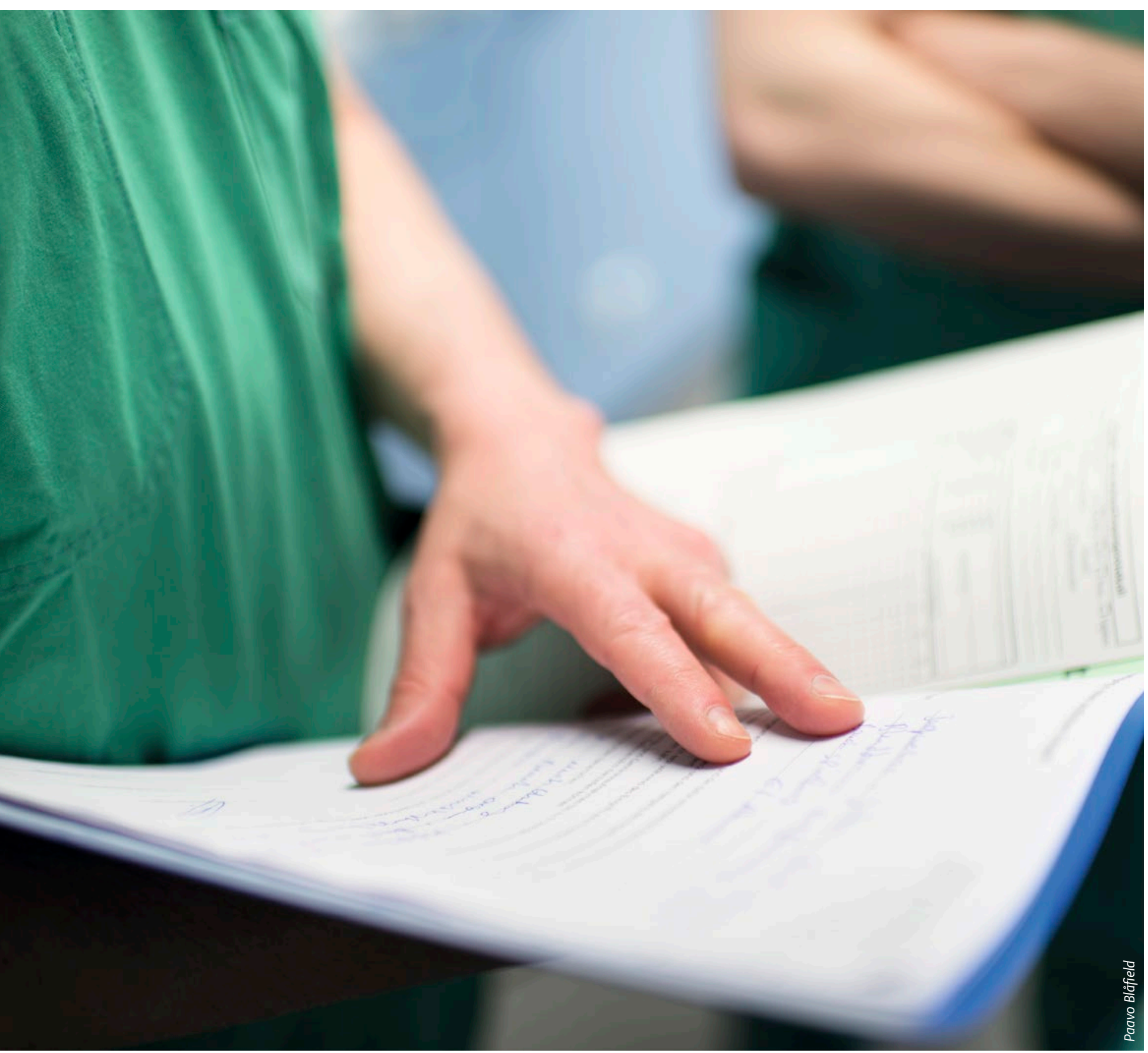


- Thomas Wytrieckus, Uwe von Hagen, Hans-Günther Henrich, Stev Langner, Conny Matthes, Melanie Schneider, Achim Schugt, Gisela Schwab -

Die personelle Ausstattung der Operationssäle in deutschen Krankenhäusern ob mit Anästhesie- oder OP-Personal steht immer wieder in der Diskussion. Das Thema füllt bereits große Anteile der OPManagementkongresse und sorgt immer wieder für Schlagzeilen.

In einer Pressemitteilung der Charité vom 28. April 2017 wurde eine Einigung zwischen der Charité und ver.di bekanntgegeben. Es werden dort nach jahrelangem Tarifstreit erstmals Mindestbesetzungen für die Bereiche Intensivpflege, Kinder- und allgemeine Pflege, Funktionspflege im OP und Endoskopie festgelegt ${ }^{1}$.

Das ist auch dringend nötig, da sich eine Unterbesetzung auch auf die Gesundheit der Mitarbeiter auswirken kann. Im Gesundheitsreport Pflege der AOK Bayern werden hohe Krankenstände der Pflegenden im Gesundheitswesen nachgewiesen. Mit durchschnittlich 22 Arbeitstagen ist diese Berufsgruppe gegenüber allen anderen am längsten krank ${ }^{2}$.

Ein Blick über den Tellerrand zeigt, dass es auch anders geht: Die erst kürzlich veröffentlichte Studie Nurse-to-Patient-Ratios ( $\rightarrow$ Info Studien) der Hans-Böckler-Stiftung zeigt, dass in einigen Ländern, wie in den USA und Australien, das Thema personelle Mindestbesetzung stellenweise mit gesetzlichen Vorgaben versehen ist beziehungsweise in den tariflichen Vereinbarungen festgelegt wurde.

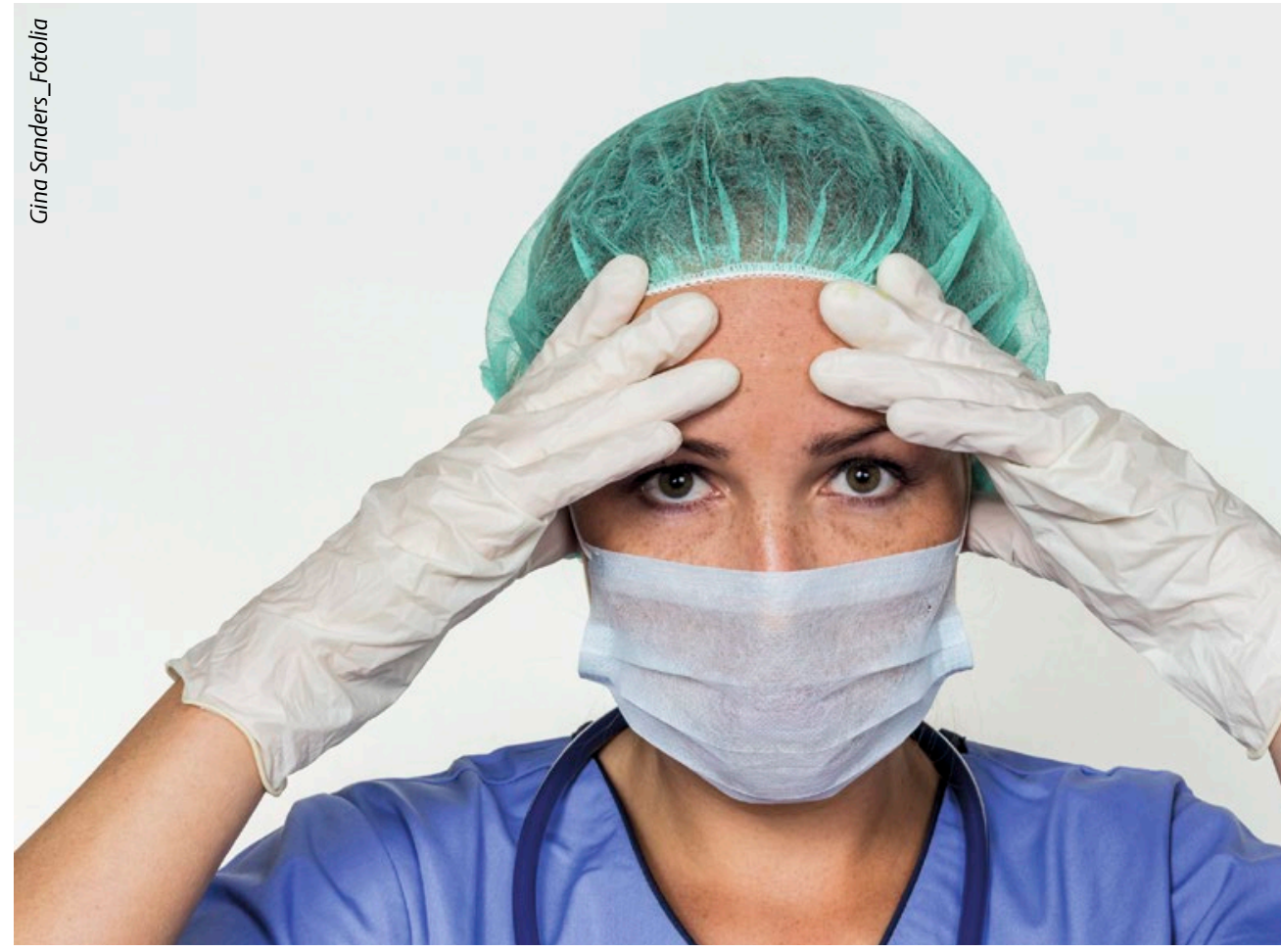

Dauerhafte Unterbesetzung kann sich auf die Gesundheit der Mitarbeiter auswirken (Symbolbild).

\section{Verbesserte Effizienz?}

In den letzten Jahren versuchen sich die Krankenhäuser weiter zu konsolidieren. Die OP-Zahlen steigen weiter rasant an. Bereits 2013 wurden 15,8 Millionen Operationen in deutschen Krankenhäusern geleistet, rund 30 Prozent mehr als im Jahr 2005.

Der Trend, die Verweildauer zu reduzieren, kombiniert mit einer Steigerung der Fallzahlen in den deutschen Krankenhäusern geht weiter, was ökonomisch und pro- zessual auf den ersten Blick für eine verbesserte Effizienz spricht.

Die Belegungstage bei steigenden Fallzahlen sind seit 2006 auf gleichbleibendem Niveau und die Zahl der aufgestellten Betten bleibt auf dem Niveau von 2006 stehen beziehungsweise senkt sich weiter $a b$.

Der Aufwärtstrend hinsichtlich der Fallzahlen scheint weiterzugehen, jedoch wird der Anstieg nach Meinung von Experten wahrscheinlich moderater ausfallen.

\section{Studien}

\section{Nurse-to-Patient-Ratios}

Bereits 2004 verabschiedete Kalifornien ein Gesetz zu einem Mindestbesetzungsverhältnis Pflege/Patient im Bereich der Intensivpflege. In dieser Veröffentlichung stellte sich heraus, dass Berufserkrankungen, die durch Überbelastung der Mitarbeiter entstehen, reduziert werden konnten ${ }^{3}$. In einer Veröffentlichung von Shekelle von 2013 wurden vorteilhafte Auswirkungen auf die Patientensicherheit im Krankenhaus untersucht. Eine der häufigsten Auswirkungen auf Patientensicherheit und Outcome ist die Personalquote in der Pflege. In mehreren Studien wurde nachgewiesen, dass es einen Zusammenhang zwischen einer geringen personellen Besetzung in der Pflege und der Steigerung von Komplikationen (zum Beispiel krankheitsbedingte Pneumonien, Dekubiti und Harnwegsinfektionen) gibt ${ }^{4}$.

\section{RN4Cast-Studie}

Die internationale RN4Cast-Studie, die von der TU Berlin für Deutschland durchgeführt wurde, zeigt eindrucksvoll die Wechselwirkungen personeller Besetzungsgrößen sowohl auf das Patienten-Outcome als auch auf das Pflegepersonal und dessen Arbeitsumgebung. Münsteraner Erklärung

Für den OP beziehungsweise die Anästhesie gibt es bereits eine Festlegung unter personellen qualitativen und quantitativen Aspekten. In der „Münsteraner Erklärung“ wird eine Besetzung von einem Anästhesisten pro OP-Saal festgelegt. Dabei gilt die komplette Narkoseführung als ärztliche Leistung, die unter Facharztstandard zu erbringen ist. ${ }^{5}$ 
Auch bei den Leistungserbringern, den Krankenhäusern, hat sich einiges verändert. Während es 2005 noch 2139 Krankenhäuser gab, waren es 2013 nur noch $2017^{6}$. Das entspricht einer Reduktion von 5,7 Prozent.

\section{Hoher Altersdurchschnitt}

Der Altersdurchschnitt des Gesundheitspersonals ( $\rightarrow$ Abb. 1) beträgt laut Statistischem Bundesamt bei den unter 30-Jährigen nur 16 Prozent. Bei den 30- bis 39-Jährigen sind es 21 Prozent. Der Mittelbau der Altersstruktur wird von den 40- bis 59-Jährigen gebildet, also rund 60 Prozent.

Prospektiv bedeutet dies, dass die Nachwuchsarbeit in der Fläche nicht mehr ausreichen könnte, um den Personalbedarf bis 2025 zu decken. Krankenhäuser, die bereits jetzt schon einen Altersdurchschnitt im OP von 48 und mehr
Jahren vorweisen, werden in den nächsten zehn Jahren auch bei einer Fluktuationsquote von zwei Prozent Probleme in der Nachwuchsbesetzung bekommen.

Diese Daten korrelieren im Hinblick auf noch zu besetzende Stellen im Funktionsdienst OP mit den Umfrageergebnissen des Verbands für OP-Management (VOPM) und des Bundesverbands Pflegemanagement aus 20148 49 Prozent der Befragten gaben an, dass ein bis fünf Prozent ihrer Planstellen nicht besetzt sind und über 36 Prozent der Krankenhäuser eine Stellenplanunterdeckung zwischen sechs und 14 Prozent aufzeigen. Bei der Anästhesiepflege ist der Personalmangel in der Umfrage jedoch deutlich geringer ausgefallen. Da ein Stellenplan für die Funktionsdienste keine personelle „Wohlfühlzone“ darstellt, kündigt ein Defizit von über drei Prozent je nach Planstellengröße schon Probleme an.

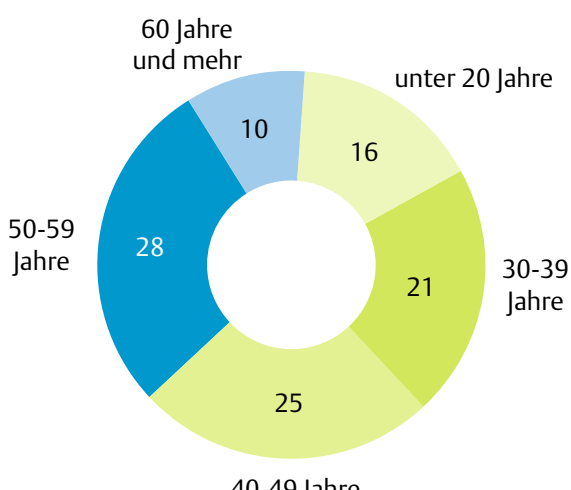

Abb. 1 Gesundheitspersonal nach Altersgruppen 2015 (nach Statistisches Bundesamt Destatis $2017^{7}$ ).

\section{Leitfaden für den OP}

Aus diesem Kontext heraus hat die Arbeitsgruppe Personal, die sich aus den Mitgliedern des VOPM und dem Bundesverband Pflegemanagement zusammensetzt, das

\section{Gemeinsamen Stellungnahme der Arbeitsgruppe Personal des Berufsverbands VOPM und des Bundesverbands}

\section{Pflegemanagement}

Regelbesetzung in den OP-Bereichen von Krankenhäusern sowie Risikobewertung von personell quantitativen Mindeststandards und qualitativen Voraussetzungen für das Personal der Funktionsdienste Anästhesie und $O P$

\section{Betrachtung der rechtlichen Aspekte im Kontext}

Im rechtlichen Kontext ist der/die Mitarbeiter/in des Funktionsbereichs Anästhesie und OP als Erfüllungsgehilfe des Arztes im Rahmen der Erfüllung eines Behandlungsvertrags zu sehen ${ }^{9}$. Das Pflegepersonal wird dabei als Helfer für den Arzt für die Umsetzung einer ärztlichen Leistung gesehen. Das Pflegepersonal und das ärztliche Personal sind im Sinne eines personellen beziehungsweise organisatorischen Über- und Unterstellungsverhältnisses verbunden ${ }^{10}$. Der Träger des Krankenhauses hat aufgrund seiner Fürsorgepflicht auf eine ausreichende Anzahl von qualifizierten ärztlichen und nichtärztlichen Mitarbeitern zu achten, die einem fachärztlichen beziehungsweise fachpflegerischen Standard entsprechen ${ }^{11}$.

\section{Qualifikationsvorgaben der Funktionsdienste im OP}

Die Mitarbeiter in den Funktionsdiensten Anästhesie und OPPflege müssen eine formelle Qualifikation nachweisen, das heißt eine abgeschlossene Aus- und Fortbildung. Dies ist dem hochkomplexen, medizinischen Bereich des OPs geschuldet.

Der Nachweis einer Qualifikation lässt die Annahme zu, dass das Personal über Wissen und Erfahrung verfügt, um in diesem Bereich arbeiten zu können. Hier sind unter anderem die Weiterbildungsrichtlinien der Deutschen Krankenhausgesellschaft (DKG) für OPPflegepersonal, die OTA/ATA-Weiterbildungsrichtlinien sowie der Leitfaden des Verbands der Pflegedirektorinnen und -direktoren der Unikliniken (VPU) zu nennen ${ }^{12}$. Durch qualifikationserweiternde Maßnahmen kann das Personal persönliche, soziale und fachliche Kompetenzen erhalten und verbessern. Dazu gehören interne beziehungsweise externe Fort- und Weiterbildungen.
Diese werden unterschiedlich vorgenommen und durchgeführt ${ }^{13}$. In dem Bereich der strukturierten Weiterführung der Wissenserweiterung und Weiterentwicklung gibt es keine Vorgaben. Aus Sicht der Arbeitsgruppe Personal ist die Förderung von Qualifikationen und deren Weiterentwicklung unumgänglich, sowohl als personalfördernde und qualitative Maßnahmen im Rahmen der Personalentwicklung als auch als Identifikations- und CorporateMerkmale des Krankenhauses beziehungsweise des Trägers.

Festlegung von personellen Bemessungsgrößen für den OP-Saal Mindestbesetzung Funktionsdienst OP

Eine Regelbesetzung für einen OP-Saal im Krankenhaus muss mit zwei Mitarbeitern des Funktionsdienstes OP angenommen werden. Dabei hängt die Qualifikationsanforderung des Personals maßgeblich von den durchzuführenden Operationen sowie den technischen, räumlichen und organisatorischen Gegebenheiten ab. Als qualifiziertes Personal ist Gesundheits- und Krankenpflegepersonal insbesondere mit entsprechender Weiterbildung sowie ausgebildetes Personal im Rahmen der OTA-Ausbildung zu sehen. Weiterhin müssen diese den ärztlichen Anforderungen (Qualifikation und Leistung) im Sinne der ärztlichen Leistungserbringung sowie der Patientenorientierung und Sicherheit Rechnung tragen. Ein zielorientierter, zeitlich wiederkehrender Abgleich von Anspruch und Leistung im gegenseitigen Dialog ist ein wirksames Mittel der beruflichen und aufgabenorientierten Verständigung. Die regelhafte Besetzung eines OP-Saals unter zwei Mitarbeitern im Funktionsdienst OP ist nur dann argumentierbar, wenn folgende Bedingungen vorliegen:

- Das OP-Portfolio lässt ein alleiniges und eigenständiges Arbeiten im Rahmen der OP-Pflegetätigkeit in einem OP-Saal zu. Eine dadurch mögliche Prozessverzögerung zum Nachteil des Patienten beziehungsweise eine hohe Arbeitsverdichtung für 


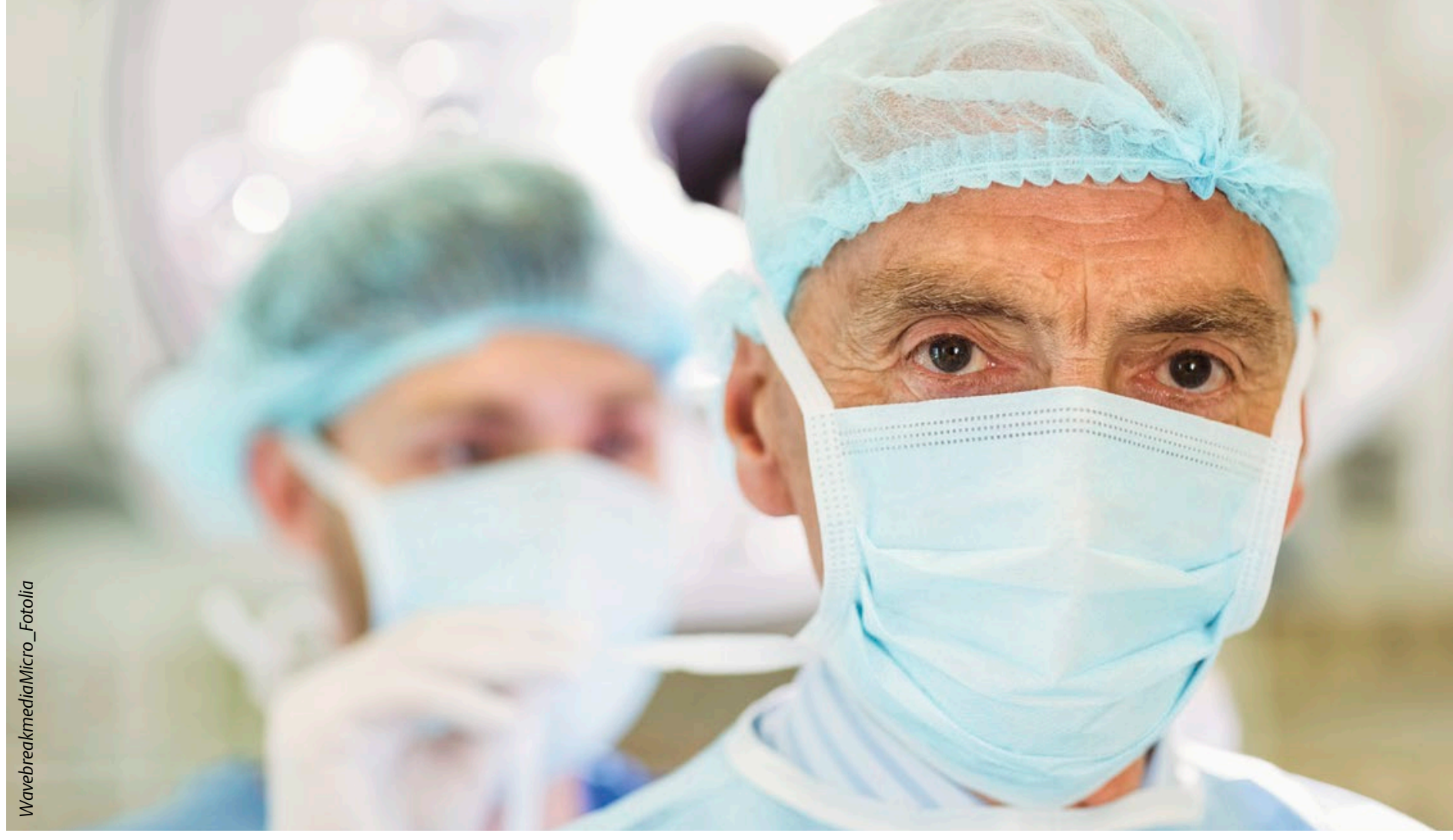

Der demografische Wandel wird in den nächsten Jahren die Regelbesetzung zusätzlich verschärfen (Symbolbild).

das Personal muss im Sinne des Zuverlässigkeitsgebots und der Risikoabwägung vermieden werden.

- Eine Risikobewertung muss belegen, dass der Umfang der Tätigkeiten des Funktionsdienstes OP zu keinem Zeitpunkt patientengefährdende Komponenten enthält.

- Der Operateur muss von der Unterschreitung der Regelbesetzung in Kenntnis gesetzt werden und sein Einverständnis geben. Er trägt gemäß der derzeitigen Rechtsprechung die Verantwortung.

- Eine weitere Fachkraft muss im Notfall unmittelbar erreichbar und verfügbar sein. Der/die alleinige Mitarbeiter/in des Funktionsdienstes OP muss die Möglichkeit erhalten, jederzeit und schnellstmöglich eine weitere Fachkraft zur Hilfestellung zu erhalten.

- Die Organisationsverantwortung liegt in der Zuständigkeit des Krankenhauses beziehungsweise des Trägers. Die Organisations- und Durchführungsverantwortung liegt bei den verantwortlichen Führungspersonen. Dabei tragen diese eine hohe Verantwortung im Bereich der Ablauf- und Arbeitsorganisation sowie in der Arbeitsüberwachung ihres nachgeordneten Personals. Daraus entstandene Fehler können haftungsrechtliche Relevanzen nach sich ziehen ${ }^{14}$.

\section{Mindestbesetzung Funktionsdienst Anästhesie}

Eine Regelbesetzung für einen OP-Saal im Krankenhaus muss mit einem Mitarbeiter des Funktionsdienstes Anästhesie angenommen werden. Als qualifiziertes Personal ist Gesundheits- und Krankenpflegepersonal insbesondere mit entsprechender Weiterbildung sowie ausgebildetes Personal im Rahmen der ATA-Ausbildung zu sehen. Weiterhin müssen diese den ärztlichen Anforderungen (Qualifikation und Leistung) im Sinne der ärztlichen Leistungserbringung sowie der Patientenorientierung und Sicherheit Rechnung tragen. Ein zielorientierter, zeitlich wiederkehrender Abgleich von Anspruch und Leistung im gegenseitigen Dialog ist ein wirksames Mittel der beruflichen und aufgabenorientierten Verständigung.
Die Regelbesetzungen unter einem Mitarbeiter im Funktionsdienst Anästhesie ist nur dann argumentierbar, wenn folgende Bedingungen vorliegen:

- Das OP- und Anästhesie-Portfolio lässt ein alleiniges und eigenständiges Arbeiten (zum Beispiel hoher Anteil von Regionalanästhesien) im Rahmen der Anästhesiepflegetätigkeit zu. Eine Prozessverzögerung zum Nachteil des Patienten beziehungsweise Arbeitsverdichtung für das Personal muss im Sinne des Zuverlässigkeitsgebots und der Risikoabwägung vermieden werden.

- Die Risikobewertung muss belegen, dass die Tätigkeit des Funktionsdienstes Anästhesie zu keinem Zeitpunkt patientengefährdende Eigenschaften enthält. Diese Risikobewertung muss allen Beteiligten vorliegen und in einem festgelegten zeitlichen Rahmen wiederholt werden.

- Der Anästhesist muss über die Unterschreitung der Regelbesetzung informiert werden und sein Einverständnis geben. Dieser trägt gemäß der derzeitigen Rechtsprechung die Verantwortung für die Durchführung der Anästhesie.

- Eine weitere Fachkraft muss bei Unterschreitung der Regelbesetzung im Notfall unmittelbar erreichbar sein. Der/die alleinige Mitarbeiter/in des Funktionsdienstes Anästhesie muss die Möglichkeit erhalten, jederzeit und schnellstmöglich eine weitere Fachkraft zur Hilfestellung zu erhalten.

- Die Verantwortung der Einsatzmöglichkeit liegt in der vollen Zuständigkeit des Krankenhauses beziehungsweise des Trägers. Die Organisations- und Durchführungsverantwortung liegt bei den verantwortlichen Führungspersonen. Dabei tragen diese eine hohe Verantwortung im Bereich der Ablauf- und Arbeitsorganisation sowie Arbeitsüberwachung ihres nachgeordneten Personals. Daraus entstandene Fehler können haftungsrechtliche Relevanzen nach sich ziehen ${ }^{14}$. 
Thema bearbeitet und diskutiert. Die Stellungnahme $(\rightarrow$ Info) dient als Leitfaden für eine verantwortungsvolle und zielgerichtete Personalbesetzung für den OP-Saal.

Neben der personellen quantitativen Besetzung muss nach Meinung der Autoren grundsätzlich die Qualifizierung in diesem Bereich weiter gefördert und ausgebaut werden. In der Umfrage der Arbeitsgruppen von 2014 investierten 37 Prozent aller Befragten nur 30 Minuten pro Monat in Fort- und Weiterbildungen des Funktionsdienstes OP und Anästhesie. 39 Prozent der Befragten können mehr als zwei Stunden pro Monat angeben. 17 Prozent der Teilnehmer führen Fortbildungen situativ aus ${ }^{8}$.

\section{Ausblick}

Bei aller Problematik der Besetzung bei den Funktionsdiensten in der Anästhesie und im OP ist die Diskussion um eine Mindestbesetzung unumgänglich. Der demografische Wandel und der Fachkräftemangel werden in den nächsten Jahren diese Entwicklung weiter zusätzlich verschärfen.

Die Auswirkungen der personellen Mindestanforderung zwingen allerdings auch die Krankenhäuser, sich thematisch intensiver mit prozessualen Möglichkeiten und Verbesserungen auseinanderzusetzen. Es gibt durchaus Möglichkeiten, in einigen operativen Fächern und ausgesuchten Operationsportfolios personell reduzierter beziehungsweise in Zusammenarbeit mit den Funktionsdiensten Anästhesie und OP zu arbeiten.

Eine strukturierte OP-Planung und Umsetzung kann diese Möglichkeiten freisetzen. Jedoch muss die geforderte $\mathrm{Ri}$ sikobewertung vom Krankenhausträger beziehungsweise den Verantwortlichen für den OP erstellt und verantwortet werden, um auch klar darzulegen, dass die etablierte Struktur möglichst umfänglich Gefährdungspotenziale in diesem Zusammenhang ausschließen kann.

Die Frage ist, ob die vorhandenen OTA/ ATA und Krankenpflegeschulen die nötige Anzahl von Mitarbeitern in Zukunft bereitstellen können, um die in die Jahre gekommenen „Babyboomer-Jahrgänge“ abzulösen. Falls nicht - und davon muss ausgegangen werden - müssen andere berufliche Grundvoraussetzungen und Aus- bildungen in die Diskussion eingebracht werden.

Im Hier und Jetzt bleibt jedoch die Erkenntnis, im Sinne einer qualitativen Patientenversorgung eine Regelbesetzungsgrundlage festzulegen und für die Qualifikation der einzelnen Mitarbeiter Sorge zu tragen. In diesem Zusammenhang ist auch das Thema Mitarbeiterzufriedenheit zu nennen. Die Umsetzung qualifizierter Fort- und Weiterbildungsmaßnahmen sowie auch Übernahme und Partizipation verschiedener fachlicher und organisatorischer Aufgaben und Verantwortlichkeiten können Motivationsfaktoren sein und das Berufsbild in den Funktionsdiensten wieder attraktiver gestalten.

Bereits jetzt schon werden in einigen OP-Bereichen Medizinische Fachangestellte (MFA) beschäftigt. Spezialisierungen, wie der Hybrid-Techniker, werden dem OP der Zukunft neue Impulse geben. Auch die Industrie ist gefordert, mehr personalunterstützende Möglichkeiten (zum Beispiel durch einfache Bedienungselemente, einheitliche Tastaturnomenklaturen, InHouse-Begleitungen) für den Arbeitsplatz OP zu entwickeln.

Die Zukunftsmöglichkeiten der operativen Bereiche werden sich maßgeblich an der medizinisch-technischen Entwicklung und den personell-prozessualen Möglichkeiten orientieren. Die Teilhabe und aktive Integration der Funktionsdienste an diesen Herausforderungen sind ein wesentliches Element in dem Prozess. Aus Sicht der Autoren stehen wir hier erst am Anfang.

\section{Literatur}

1 Charité. Tarifkonflikt zur Pflegepersonalausstattung beigelegt. Pressemitteilung der Charité vom 28.04.2017. Im Internet: https://www.charite.de/ service/pressemitteilung/artikel/detail/einigung_ zwischen_charite_und_verdi/; Stand: 02.08.2017

2 RZH. DAK und AOK melden hohen Krankenstand im Gesundheitswesen. Im Internet: https://www. rzh.de/service/branchen-news/dak-und-aok-melden-hohen-krankenstand-im-gesundheitswesen; Stand: 02.08.2017

3 Mark B, Harless D, Spetz J et al, California`s Minimum Nurse Staffing: Results from a Natural Experiment. HSR Healt Serv Res v 48 (2 PT 1); 2013 Apr PMC 3626342

4 Shekelle P. Effect of Nurse-to-Patient Staffing Ratios on Patient Morbidity an Mortality in Making Health Care Safer II; An Updated Critical Analysis of the Evidence for Patient Safety Practices. Evidence Reports/Technology Assesments No. 211; Mar 2013
5 Gemeinsame Stellungnahme des Berufsverbandes Deutscher Anästhesisten und der Deutschen Gesellschaft für Anästhesiologie und Intensivmedizin. Anästhesiol Intensivmed Notfallmed Schmerzther 2005; 40 (1): 4-5

6 Statistisches Bundesamt. Anzahl der Krankenhäuser in Deutschland. Im Internet: https:// de.statista.com/statistik/daten/studie/2617/umfrage/anzahl-der-krankenhaeuser-in-deutschland-seit-2000/; Stand: 02.08.2017

7 Statistisches Bundesamt. Gesamtkatalog 2017. Im Internet: https://www.destatis.de/DE/Publikationen/Gesamtkatalog.html;jsessionid=E83D1 5091BB3607B30213C6B83BF8DCE.cae2; Stand: 02.08.2017

8 Loges-Fechtner B, Hagen U, Henrich HG, Langner S, Schwab G, Wytrieckus T. Ein weiter Weg. Im OP 2016; 06 (03): 129-135

9 Vgl. §§276, 278, 280 BGB

10 Vgl. Sträßner HR. Rechtliche Probleme in der Operationspflege. Pflegerecht 2012; 1: 1

11 Vgl. Bergmann VersR; 1996: 810, 812

12 Vgl. Sträßner HR. Rechtliche Probleme in der Operationspflege. Pflegerecht 2012; 1: 3

13 Vgl. Blum K, Löffert S, Offermanns M. et al. DKI Krankenhausbarometer; 2013: 88 ff

$14 \mathrm{Vgl}$. Sträßner HR. Rechtliche Probleme in der Operationspflege. Pflegerecht 2012; 1: 12

\section{Autoren}

Uwe von Hagen

OP-Manager Stadtkrankenhaus Korbach

Hans-Günther Henrich

OP-Manager Klinikum Fulda

\section{Stev Langner}

OP Manager Klinikum Brandenburg

\section{Conny Matthes}

Funktionsdienstleitung Zentrales OP-Management, Universitätsmedizin Mainz

\section{Melanie Schneider}

Pflegedienstleitung St. Christophorus Krankenhaus Werne

\section{Achim Schugt}

OP-Manager Marienhospital Stuttgart

\section{Gisela Schwab}

OP-Leitung Leopoldina Krankenhaus Schweinfurt

Thomas Wytrieckus

OP-Manager Maria Hilf Kliniken Mönchengladbach E-Mail: Thomas.Wytrieckus@mariahilf.de

\section{Bibliografie}

DOI 10.1055/s-0043-116997 Im OP 2017; 7: 254-258

(c) Georg Thieme Verlag KC

Stuttgart · New York · ISSN 1611-7905 